\title{
Criminologie
}

\section{La surveillance de la liberté et la pratique criminologique}

\section{Yvon Dandurand et Jacques Laplante}

Volume 12, numéro 2, 1979

Probation : aide ou contrainte?

URI : https://id.erudit.org/iderudit/017109ar

DOI : https://doi.org/10.7202/017109ar

Aller au sommaire du numéro

Éditeur(s)

Les Presses de l'Université de Montréal

ISSN

0316-0041 (imprimé)

1492-1367 (numérique)

Découvrir la revue

Citer cet article

Dandurand, Y. \& Laplante, J. (1979). La surveillance de la liberté et la pratique criminologique. Criminologie, 12(2), 78-88. https://doi.org/10.7202/017109ar d'utilisation que vous pouvez consulter en ligne.

https://apropos.erudit.org/fr/usagers/politique-dutilisation/ 


\section{LA SURVEILLANCE DE LA LIBERTÉ ET LA PRATIQUE CRIMINOLOGIQUE}

Yvon Dandurand * Jacques Laplante

L'agent de surveillance s'est trouvé face à une tâche de plus en plus difficile au cours de ces dernières années. La pratique criminologique et les différentes façons de la concevoir en ont également été affectées. Les contradictions et la confusion de plus en plus manifestes dans les objectifs pénaux et correctionnels ont, semble-t-il, entraîné les responsables de la liberté surveillée dans des difficultés quasi insolubles. La belle époque des années soixante semble prendre brutalement fin; l'idéal de la réhabilitation du criminel est durement attaqué et il menace d'entraîner dans sa chute non seulement une certaine conception de la liberté surveillée, comme outil d'intervention, mais également tout le système de liberté surveillée qui a eu la malchance d'être trop étroitement lié au modèle médical d'intervention. Le concept même d'intervention pénale ou correctionnelle est en proie à un vaste exercice de redéfinition. Le résultat de cet exercice ne peut se solder que par la remise en cause du principe même de la liberté surveillée.

La mesure se trouve, pour ainsi dire, à une sorte de croisée des chemins. Or, l'on ne peut évidemment pas dissocier la question de l'avenir du système de liberté surveillée de celle, plus gobale, de l'avenir de l'intervention pénale. Les propositions de réforme concernant l'ensemble du système correctionnel, de retour à des sentences déterminées et de l'élimination à plus ou moins court terme de la libération conditionnelle, ou encore telles que suggérées comme des alternatives à l'emprisonnement, entraînent toutes des répercussions considérables sur le concept et le système de liberté surveillée. Toutefois, ce qui nous apparait encore plus important dans la situation actuelle, c'est que ces différentes propositions sont toutes avancées au moment même où la pratique criminologique, à la suite de la déroute du modèle médical d'intervention et de l'idéal de la réhabilitation du criminel, se trouve elle-même dans un état de profonde confusion.

* Y. Dandurand et J. Laplante sont professeurs au Département de criminologie à l'Université d'Ottawa. 
Nous croyons que l'avenir de la liberté surveillée, en tant que concept qu'en tant que système, dépendra largement des agents de surveillance et de leur capacité de définir pour euxmêmes une pratique criminologique plus réaliste, c'est-à-dire une pratique qui tienne compte véritablement de la nature intrinséquement politique du phénomène criminel et de l'intervention pénale.

\section{NÉCESSAIRE RÉINVENTION}

\section{DE LA PRATIQUE CRIMINOLOGIQUE}

Aujourd'hui, le modèle médical d'intervention est soumis à une critique radicale, à une remise en question fondamentale. L'idéal de la « réhabilitation du criminel s est tombé en désuétude et le déclin du modèle médical d'intervention face au crime va en s'accélérant. Les différents aspects du système d'administration de la justice qui sont apparus comme des conséquences de l'application de ce modèle d'intervention sont remis en question et quelquefois menacés purement et simplement d'abolition par rapport a leur fonction traditionnelle; c'est le cas, par exemple, des services correctionnels tels que la probation ou la libération conditionnelle.

Les opposants du modèle d'intervention et, en même temps, d'une pratique criminologique se réclamant de ce modèle sont nombreux et ils rencontrent un auditoire de plus en plus disposé à les écouter. Ces personnes appuient leurs critiques sur ce qu'elles affichent comme étant un réalisme nouveau ou renouvelé. L'on prête déjà à cette cécole de pensée , le nom de "new realists $>$. Ce que ces nouveaux réalistes proposent, c'est une sorte de retour à une tradition et à des modèles d'intervention qui ne diffèrent que très peu de celui proposé il y a longtemps par l'école classique de criminologie, modèle reposant principalement sur la notion de libre arbitre et mettant l'emphase sur la responsabilite du criminel (MacNamara, 1977 ; Schrag, 1977, Fattah, 1978).

Mais en quoi consiste une pratique criminologique réaliste ? Comment peut-on définir une intervention qui ne représente pas un retour en arrière et qui ne sacrifie pas inutilement les quelques progrès humanitaires que le modèle médical a permis de

1. Revie Crime and Social Justice, no 8, Editorial. 
réaliser ? Ou, comme Fattah (1978) posait la question, est-il nécessaire de sacrifier l'idéal humanitaire pour accéder à une pratique criminologique plus efficace?

Il est clair que la criminologie, et spécialement la criminologie pratique, a connu des succès et s'est développée à un rythme rapide en partie, sinon uniquement, parce qu'elle representait l'instrument par lequel le modèle médical pouvait être implanté rapidement à travers le système canadien de justice pénale. (Voir : Szabo, 1977 ; Dandurand, 1976 ; Bertrand, 1975). Le criminologue s'est trouvé médecin malgré lui pour toutes sortes de raisons dont certaines moins avouables que d'autres.

Le praticien peut s'attacher au modèle médical et, peut-être, sombrer avec ce dernier; il peut aussi retourner à des bases plus solides, plus conformes aux enseignements d'une criminologie non clinique.

En effet, les criminologues ont, semble-t-il, ignoré la plupart des enseignements importants de la sociologie criminelle et de la sociologie du droit. La criminologie pratique continue de limiter l'usage qu'elle fait des enseignements de la sociologie aux théories sociologiques primitives de la déviance (théories des facteurs sociaux d'inadaptation), aux théories de la tradition analytique-individuelle, aux théories qui ne remettent pas en question l'idée que le criminel est nécessairement un individu anormal, différent. La criminologie pratique a refusé de reconnaître que tout le phénomène de contrôle social est un phénomène politique.

De fait, il est aisé de constater que la criminologie pratique a systématiquement éliminé de ses préoccupations et de ce qu'elle retenait de la criminologie théorique tout ce qui n'était pas réconciliable avec l'idéologie du modèe médical auquel elle souscrivait : ce fut une condition sine qua non de son développement. D'ailleurs, les criminologues ont été critiqués à maintes reprises pour cette position (Bertrand, 1973 ; McNaughtonSmith, 1973 ; 1976). Certains criminologues, réussissaient de temps à autre à prendre une certaine distance par rapport à ce modèle et à l'idéologie qui le sous-tendait. Par contre, ceux qui avaient à travailler à l'intérieur d'un système conçu en fonction de l'application de ce modèle, ceux qui acceptaient le rôle de emédecin s (et le prestige social qui en découlait) n'avaient 
véritablement d'autre choix que de continuer à souscrire à ce modèle. Les praticiens étaient très souvent sur la défensive, mieux informés que quiconque des limites concrètes du modèle auquel ils souscrivaient et mal à l'aise lorsqu'il s'agissait de le défendre.

Il est possible de définir le rôle de la pratique criminologique autrement que de l'intérieur du modèle de réhabilitation. De toute façon, il semble de plus en plus probable que le criminologue praticien n'aura pas le choix. Son rôle de médecin va lui être retiré malgré lui comme lorsqu'il lui avait été conféré.

\section{DIFFERENTS SCENARIOS POSSIBLES}

Dans quel contexte cette redéfinition de la pratique criminologique, dans le cadre de la surveillance de la liberté des contrevenants, est-elle susceptible de s'effectuer? Plusieurs scénarios sont possibles et il peut s'avérer utile de les examiner un peu plus attentivement. Nous en considérerons trois brièvement :

1. celui du maintien des tendances actuelles;

2. celui de la possibilité d'assister à une réforme importante du système d'administration de la justice ;

3. celui qui ferait suite à l'abolition des prisons ou d'autres parties complètes du système d'administration de la justice.

\section{Le maintien des tendances actuelles}

En admettant que les tendances actuelles soient maintenues, l'on s'attendra à une intensification de la lutte contre l'emprisonnement et de l'opposition au modèle traditionnel de traitement en liberté surveillée. Les efforts seront sans doute placés sur la participation communautaire encadré par des services officiels tels ceux de la probation et de la libération conditionnelle. Voyons dans quel contexte se présente ce scénario et les possibilités qu'il offre.

Nous savons pertinemment que le système de justice criminelle ne touche qu'une partie bien mince des contrevenants. Ceux qui ne sont ni arrêtés, ni envoyés devant les tribunaux, ni condamnés constituent un pourcentage beaucoup plus élevé de la population globale des contrevenants. Selon les estimés basés sur un échantillon national de 1973 des victimes de crimes aux 
Etats-Unis, seulement vingt-huit pour cent des crimes sont rapportés à la police (Skogan, 1977). Il est inutile de dire que nous ignorons tout du contrevenant non arrêté.

Ce que l'on sait des criminels est entièrement limité aux personnes arrêtées, envoyées au tribunal et condamnées. Les délinquants ont ainsi toutes les chances d'être membre de la classe sociale et économique la plus défavorisée, d'avoir une mauvaise éducation et de provenir d'un foyer peu structuré (Commission Katzenbach, 1967, Commission d'enquête Prévost, 1968).

Nous faisons une ségrégation; elle est évidente! Mais ce n'est pas tout; s'ajoutent à la ségrégation les coûts de cette dernière, l'évidence de l'usage excessif des prisons et la certitude que la détention n'enraye pas davantage la récidive que les autres moyens correctionnels telle la liberté surveillé (Robinson et Smith, 1971 ; Sinclair, 1971 ; Martinson, 1971; Wilkins, 1969). Comme la liberté surveillée est moins coûteuse, plus humaine et d'efficacité égale, il va de soi que les réformistes veuillent l'intensifier.

La contestation des prisons puis des modeles traditionnels de liberté surveillée a donné lieu à une volonté de retourner aux sources et de refaire le contact avec la communauté afin de combler le fossé entre le criminel et les autres citoyens. Nous assistons dorénavant à une ruée vers les programmes communautaires qu'on retrouve sous diverses appellations dont la déjudiciarisation, la médiation, la restitution, les ordonnances de travaux communautaires ou les centres résidentiels (Laplante, 1978).

Les expériences menées dans ces domaines n'apportent; a part une dimension plus humaine que la prison et un recul très partiel du modèle médical, qu'un changement fort limité aux méthodes correctionnelles. Plus encore certains de ces programmes supposés innovateurs comme la déjudiciarisation (Laplante, 1977) se sont avérés comme accélérant la ségrégation auquel nous faisions allusion.

Nous sommes forcés de noter que le processus politique et organisationnel, propre à changer les services correctionnels, nécessite des changements profonds et intensifs sur plusieurs fronts à la fois. Nous pouvons voir comment les communautés sont 
réticentes à s'engager dans la résolution de leurs conflits (Normandeau et Léveillée, 1977) mais d'autre part il est aisé de constater les partis pris des pouvoirs en cause. Une décentralisation de l'administration de la justice est nécesaire afin d'encourager les communautés à s'occuper de leurs membres et à établir leurs propres critères pour résoudre les confiits qui surgissent chez elles. Les fonctionnaires de la justice devraient servir à aider les communautés dans ce sens, mais non pas le contraire, soit imposer les critères et demander ensuite la participation des communautés. Cette dernière façon d'agir est vouée d'avance à l'échec et conduit le fonctionnaire à blâmer la communauté.

\section{Le scénario de la réforme de l'administration de la justice}

Dans le cas du deuxième scénario possible, une lutte s'intensifie non seulement en regard de la prison et du modèle médical d'intervention, mais aussi contre l'ensemble du système de justice. La liberté surveillée et la pratique criminologique s'en trouveraient affectées d'une façon bien différente.

La Commission de réforme du droit (1976) n'hésite pas à affirmer que l'inflation législative, pénale et judiciaire menace l'appareil pénal d'effondrement total. L'idée que l'on pouvait résoudre tous les problèmes en légiférant a conduit à une prolifération de lois, de règlements et d'infractions (près de 70000 de ces dernières aux trois niveaux de gouvernement).

Ce qui est pire encore, c'est que cette tendance, conjuguée à la croyance populaire de l'efficacité du droit pénal et de l'intimidation, a conduit à une foule d'infractions de responsabilité stricte (70\% des lois fédérales et $90 \%$ des lois provinciales). Le tableau s'assombrit encore lorsque l'on constate que $70 \%$ des infractions de responsabilité stricte sont punissables par des peines de prison (dont une forte proportion des infractions à la circulation).

Cette façon que nous avons d'agir coute cher à l'usager, à ses proches et à l'ensemble des citoyens qui de plus croient que la cour va régler le problème et que les services correctionnels compléteront peut-être la tâche en réhabilitant le fautif. Une façon de combattre ces excès et d'affaiblir les mythes qui s'y accrochent peut se situer au niveau de la non-intervention et du recours aux services privés. 


\section{a) La non-intervention ou l'intervention minimale}

Fondamentalement, la non-intervention implique toute politique qui accommode la société dans la plus grande variété possible de ses comportements et de ses attitudes, plutôt que de forcer autant de personnes que possible à s'ajuster à de supposés standards sociaux communs (Schur, 1973).

Le rôle de la police, de la cour et des agents correctionnels est change drastiquement. Toutes les fois qu'ils le peuvent, les policiers, les juges et les agents correctionnels laissent le déviant tranquille. De plus, les chambres des communes restreignent leur hausse à l'inflation législative mais éliminent les infractions inutiles dont s'occupent les instances de justice.

Dans ce cas, une des fonctions principales de la pratique criminologique devient précisément de travailler à augmenter la tolérance de la communauté et de la société en général à l'égard de certains types de comportements déviants.

\section{b) Le recours au secteur privé}

Un essai a été fait au Massachusetts, il y a déjà huit ans, quand plus de 1000 jeunes se trouvaient dans les institutions de réforme. Il n'en reste actuellement que 70 dans de telles institutions alors que le système de justice du Massachusetts s'occupe d'environ 2000 jeunes à l'aide de la supervision à domicile, de programmes de jour non résidentiels, de l'aide aux familles, de foyers de groupe, de programmes en plein air (Corrections Magazine, septembre 1978). Presque tous ces programmes sont sous la responsabilité de groupes privés qui ont signé des contrats avec le ministère responsable des services à la jeunesse. Il s'est avéré que la communauté collabore davantage avec les services privés et n'acceptent pas de se laisser entraîner dans les dédales de la bureaucratie.

Au Canada, cette réforme au niveau de la non-intervention, liée ensuite à un certain degré de confiance manifestée à l'égard du secteur privé, n'est certes pas pour demain. Bien que nombre d'agents de probation et de libération conditionnelle voient clairement le bien-fondé de telles orientations, ils n'y peuvent pas grand-chose. Ils sont plutôt devenus des boucs émissaires au 
cours de ces dernières années, tenus responsables en partie de la dégradation du système et en même temps amenés ironiquement à le soutenir par des supposées innovations communautaires.

Le comité Batshaw a fait des recommandations timides sur l'intervention minimale; il y a aussi des projets de loi sur les jeunes qui ont des démêlés avec la justice où le légalisme, en voulant remplacer la bonne volonté du juge comme père, effraie tout simplement lorsque l'on repense aux excès de procédures et à leurs conséquences. Mais ces efforts louables sont contrebalancés facilement par des lois tenant automatiquement les meurtriers à l'écart pour une longue période ou par celles concernant les armes à feu, ainsi que par bien d'autres. Enfin, il est difficile de ne pas référer aux projets législatifs concernant l'obscénité et les délits d'ordre sexuel.

L'agent de liberté surveillée aura à réparer une fois de plus les pots cassés. Sera-t-il encore « médecin > malgré lui ? Est-ce que la réforme correctionnelle, intrinsèquement rattachée au système par force ou résignation, n'est qu'une illusion ? Est-ce que l'inefficacité globale et le danger de notre justice coûteuse retomberont sur les plus vulnérables des acteurs à l'intérieur du système ? (Il y a longtemps que les usagers en payent le coût). Est-ce que le mythe selon lequel la suppression de la peur du crime et du crime lui-même et l'encadrement des méchants sont des objectifs désirables et réalisables grâce au développement du système de la justice ? Est-ce que la personne a perdu à jamais sa capacité de s'adapter à son environnement et aux humains qui le composent?

\section{Le scénario abolitionniste}

Les tenants de la réforme mettront naturellement leur confiance dans un système renouvelé qui redonnerait à l'homme sa dignité. Les abolitionnistes, que ce soit des systèmes de justice et du droit pénal lui-même, diront que la réforme supposée continuera à établir une nette ségrégation entre les classes, que les institutions de justice que nous avons sont médiévales et que pour rendre à l'homme sa dignité il faut se débarrasser de ces enclaves archaìques. C'est le scénario de l'abolitionnisme que nous ne pouvons développer ici. 


\section{Conclusion}

Nous croyons qu'à court terme le criminologue engagé dans le travail de la liberté surveillée ne peut se libérer lui-même du premier scénario soit du maintien des tendances actuelles. Il doit dès lors concentrer ses énergies sur une intervention réaliste.

Lorsque les criminologues prennent conscience du caractère essentiellement politique de l'intervention criminologique, ils ont tendance dans un premier temps à rejeter le système d'administration de la justice et à prendre position pour le criminel contre les mécanismes du contrôle social. C'est une option politique radicale, qui peut sans doute être respectée, mais à laquelle très peu de criminologues peuvent souscrire, spécialement parmi les praticiens qui sont aux prises avec le quotidien du phénomène criminel, avec les plaintes provenant des victimes, avec les dommages causés par les crimes et avec les aspects socialement dommageables de la criminalité. Ils ne peuvent faire ce saut; ils ne peuvent s'identifier, dans une sorte d'élan romantique, au criminel, parce qu'ils ont conscience également des conséquences des formes traditionnelles du crime.

Le criminologue praticien se dit alors qu'il doit $y$ avoir un choix. Peut-être est-il possible d'aider celui qui est victime du système sans pour cela prendre la défense de ce système de contrôle qui est très souvent sur-répressif, arbitraire et qui permet a certaines personnes puissantes de violer impunément la personne et la propriété d'autrui et de soumettre d'autres individus à toutes sortes de dégradations sous prétexte que ces derniers représentent une menace pour la société. Le praticien ne souscrit que très rarement à l'image romantique du criminel que certains theoriciens radicaux voudraient imposer.

Ce qu'il faut, au praticien, c'est un outil conceptuel visant à définir la pratique criminologique, qui repose, tel qu'indiqué plus tôt, sur une définition de ce qui constitue la réalité fondamentale du phénomène criminel. C'est reconnaître que la donnée première du phénomène criminel, repose sur le fait que le comportement de certains individus n'est pas toléré par certains autres qui sont plus puissants et que cette intolérance se manifeste de façon criminalisante, par et à travers le système de justice pénale. Si ce fait premier est intégré dans la pratique 
criminologique, il peut remplacer le modèle d'intervention basé sur la réhabilitation, ou sur la notion que le criminel est nécessairement un individu différent des êtres dits « normaux * (uniquement parce qu'il a commis un crime) et que le but de l'intervention criminologique consiste à le rendre (normal s.

Le modèle d'intervention proposé est dit réaliste parce qu'il présuppose une confrontation permanente du praticien et du criminel à la réalité fondamentalement politique du phénomène de contrôle social. C'est un modèle qui ne permet pas à l'intervenant d'oublier que son intervention se situe à l'intérieur d'un processus politique. Comme intervenant le criminologue vise, dans ce contexte, à ce que le déviant utilise les mécanismes légaux, culturels et sociaux plutôt que de les subir.

Au niveau du deuxième scénario, soit la réforme de l'administration de la justice et à moyen terme (10-15 ans), le criminologue peut faire toutes sortes de suggestions en ce qui a trait à la décriminalisation, favoriser la non-intervention et s'engager lui-même dans le secteur privé. On compte quelques exemples éparses de ce phénomène.

Enfin, face au scénario abolitionniste, il semble que seul le criminologue praticien désengage du système peut vraiment avoir une influence à moyen terme à ce niveau. 


\section{BIBLIOGRAPHIE}

BERTRAND, M.-A. (1973) : - Dialogue with Marie-Andrée Bertrand, (an interview conducted by V.E. Grabbiner), Issues in Criminology, vol. 8 , no. 2 : 31-49.

CANADA (1976) : Notre droit pénal, Ottawa, Commission de réforme du droit.

DANDURAND, Y. (1976) : * The Professional Criminologist in Canada s, Criminologie Made In Canada, Vol. 3, No. 1 \& 2, 153-160.

FATTAH, E.A. (1978) : Moving to the Right : A Return to Punishment ? , Crime et/and Justice, vol. 6, no. 2.

LAPLANTE, J. (1977) : - La déjudiciarisation : sa portée au niveau communautaire s, Déviance et société, 4, 459-470.

LAPLANTE, J. (1978) : * Les alternatives à l'emprisonnement : la déjudiciarisation, la médiation, la restitution, les ordonnances de travaux communautaires, les centres résidentiels communautaires \#, Société canadienne pour la prévention do crime : conférence sur la participation du citoyen en matière de justice pénale.

MAC NAMARA, D.E.J. (1977) : The Medical Model in Corrections : Requiescat in Pace s, Criminology, vol. 14, no. 4. 439-448.

MAC NAUGHTON-SMITH, P. (1973) : * The Role of the Criminologist in Society >, 14e Congrès annuel de la Société de criminologie du Québec, Montréal, 15 mars 1973.

MAC NAUGHTON-SMITH, P. (1976) : La liberté au compte-gouttes, Ottawa, Commission de réforme du droit du Canada.

MARTINSON, R.M. (1971) : Treatment Evaluation Survey, New York, Division of Criminal Justice Services.

QUEBEC (1968) : La sociêté face au crime, Commission d'enquête sur l'administration de la justice en matière criminelle et pénale au Québec.

ROBINSON, J., SMITH, G. (1971) : The Effectiveness of Correctional Programs \#, Crime and Delinquency, 17, 67-80.

SCHRAG, C. (1977) : * Thinking About Crime s, e Punishing Criminals : Concerning a Very Old and Painful Question ", "We Are the Living Proof : The Justice Model for Corrections >, book reviews, Criminology, vol. 14 , no. 4, 569-573.

SCHUR E.M. (1973) : Radical Non-Intervention, New York, Spectrum.

SINCLAIR, I.A.C. (1971) : Hostels for Probationers, London, H.M.S.O.

SKOGAN, W.G. (1977) : Eimension of the Dark Figure of Unreported Crime ", Crime and Delinquency, 1, 41-50.

SZABO, D. (1977) : Histoire d'une expérience québécoise qui aurait pu mal tourner, Criminologie, vol. $\mathrm{X}$, no. 2, 5-38.

U.S.A. (1967) : The Challenge of Crime in a Free Society, President's Commission on Law Enforcement and Administration of Justice.

WILKINS, L.T. (1960) : Evaluation of Penal Measure, New York, Random House. 